A. Pramesh Rao, G. Swarup and Gopal-Krishna, eds.

\title{
On the association of G343.1-2.3 and PSR 1706-44
}

\author{
R. Dodson
}

University of Tasmania, Australia

K. Golap

NRAO, USA

J. Osborne

University of Durham, UK

N. UdayaShankar

Raman Research Institute, India

\begin{abstract}
The observations of the supernova remnant G343.1-2.3 with the Mauritius Radio Telescope, the Australia Compact Array, and the Hobart Single dish are presented. With these more sensitive measurements the association with the pulsar PSR1706-44 becomes much more likely. The major points from the observations are presented.
\end{abstract}

\section{Introduction}

The pulsar PSR B1706-44 is one of only 7 that are known to emit gamma-rays in the GeV energy range and one of only 3 that have been detected in the $\mathrm{TeV}$ range. There is thus considerable interest in the circumstances of the origin and evolution of such high energy pulsars.

The radio pulsar was discovered by Johnston et al (92). McAdam et al (93) published a map made by the MOST telescope at $843 \mathrm{MHz}$ of the area around the source. It showed a semicircular arc of emission, which has subsequently been denoted as the supernova remnant (SNR) G343.1-2.3, with the pulsar seemingly embedded in a small feature at its south eastern extremity. The $\Sigma-D$ distance is $3 \mathrm{kpc}$ for the remnant. The pulsar distance indicated by the Taylor and Cordes model was only $1.8 \mathrm{kpc}$. Frail et al (94) and Nicastro et. al. (96) both argued against the associations.

\section{The Importance of Associations}

Incorrect, or chance, associations can bias statistical results, especially when the number of objects is small. Pulsar and supernova remnant associations have been examined critically by Kaspi (96). 
This pulsar, has a characteristic age of about 17.5 kyears (Johnston et al 92), a HI absorption distance of 2.4-3.2 kpc (Korbalski 95) and a scintillation velocity of $<=100 \mathrm{kms}^{-1}$ (Dodson 97). The SNR G343.1-2.3 has an age of between 8 and 17 kyears, and a distance of 3-3.6 kpc (Dodson 97). The pulsar sits about $2 / 3^{r d s}$ from the geometric centre requiring a velocity of $750 \mathrm{kms}^{-1}$.

The distance from the Taylor and Cordes model, $1.8 \mathrm{kpc}$, is clearly in error. The values for the scintillation bandwidth to velocity have been recalculated to reflect the improved models (Dodson 97). The shortfall in the scintillation velocity is not of great concern as it is within the $2 \sigma$ limit of the distribution of measured velocity to scintillation velocity, furthermore the geometric centre is rarely the true $\mathrm{SN}$ site.

Altering Kaspi's scoring method (96) slightly, (justified in detail in Dodson 97 ), these change the previous score of -3 to one of +2 . These are summarised in table 1 .

\begin{tabular}{|l|l|l|l|l|}
\hline Age (kyr) & Distance (kpc) & $\beta$ & $v_{t}(\mathrm{~km} / \mathrm{s})$ & Score \\
\hline \multicolumn{5}{|c|}{ Previous PSR to SNR values } \\
\hline $17.5 / ?$ & $1.8 / 3$ & 1.0 & $<50 / 1000$ & -3 \\
\hline \multicolumn{5}{|c|}{ Now } \\
\hline $17.5 / 8-17$ & $2.4-3.2 / 3-3.6$ & 0.7 & $<100 / 750$ & 2 \\
\hline
\end{tabular}

Table 1. Pulsar and SNR parameters

\section{Observations of G343.1-2.3}

The Mauritius Radio Telescope (MRT) (Golap et al 98) has observed this supernova remnant at $151.5 \mathrm{MHz}$, and a resolution of $4^{\prime} \times 4^{\prime} .2$. I present preliminary results, with a resolution of $4^{\prime} \times 15^{\prime}$. The MRT is particularly suited for non-thermal (as it is low frequency) and broadscale sources (as it is a filled $u v$ instrument).

Follow-up observations were done with the Australian Telescope Compact Array (ATCA), and the Mount Pleasant 26m single dish instrument. These were combined to produce the the final maps.

These show that the observations with MOST, the ATCA and VLA are under estimating both the received flux and source extent.

\section{The Spectral Index}

The spectral index of this source is the clearest evidence of the problem with the other observations. The single dish observations show a monotonic spectral index of 0.5 However the MOST, VLA and ATCA-only observations find a significant short fall in flux and (more importantly) extent. It is only when the single dish information is included in the interferometer images that sensible results can be obtained. 

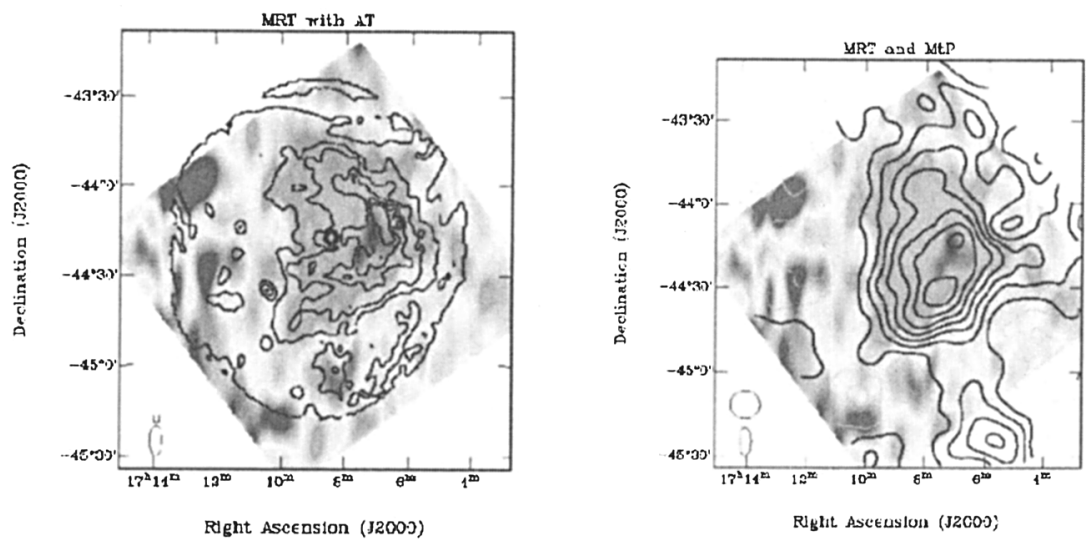

Figure 1. MRT overlayed with a) ATCA and b) Mt Pleasant

\section{Techniques for Image Recovery}

There is no replacement for making the observation. The total power is the most important measurement if one is not to under estimate the flux from and the extent of the source. This is best done with a single dish observation. For the ATCA, the dishes are $22 \mathrm{~m}$ in diameter and and a closest approach of $30 \mathrm{~m}$. Therefore the Parkes $64 \mathrm{~m}$ dish is an ideal $u v$ filler. However it is also 10 times over subscribed, so Mount Pleasant, with a diameter of $26 \mathrm{~m}$, is quite adequate.

It is gratifying to note that MRT, an interferometer, also includes the shortest possible spacing and thus recovers the broadscale structure naturally.

\section{The ROSAT X-ray Observations}

As one can see, the data is being pushed hard; the background subtraction is distorted by the LMXB 1705-44 and the poor PSF of ROSAT. Full details of how the background subtraction and model fitting were done can be found in Dodson (97). Fortuitously, the distance, but not the age, (Figure 2) is robust for the range of possible model fits to be $3-3.6 \mathrm{kpc}$. The age can only be estimated as between $8-17$ kyears.

\section{Conclusions}

The association has been shown to be highly likely, with good alignment in both time and space. The uncertainties in the X-ray model will be tackled with the new generation of X-ray observatories. The question of the pulsar proper motion will be measured as soon as we have a phase reference. 

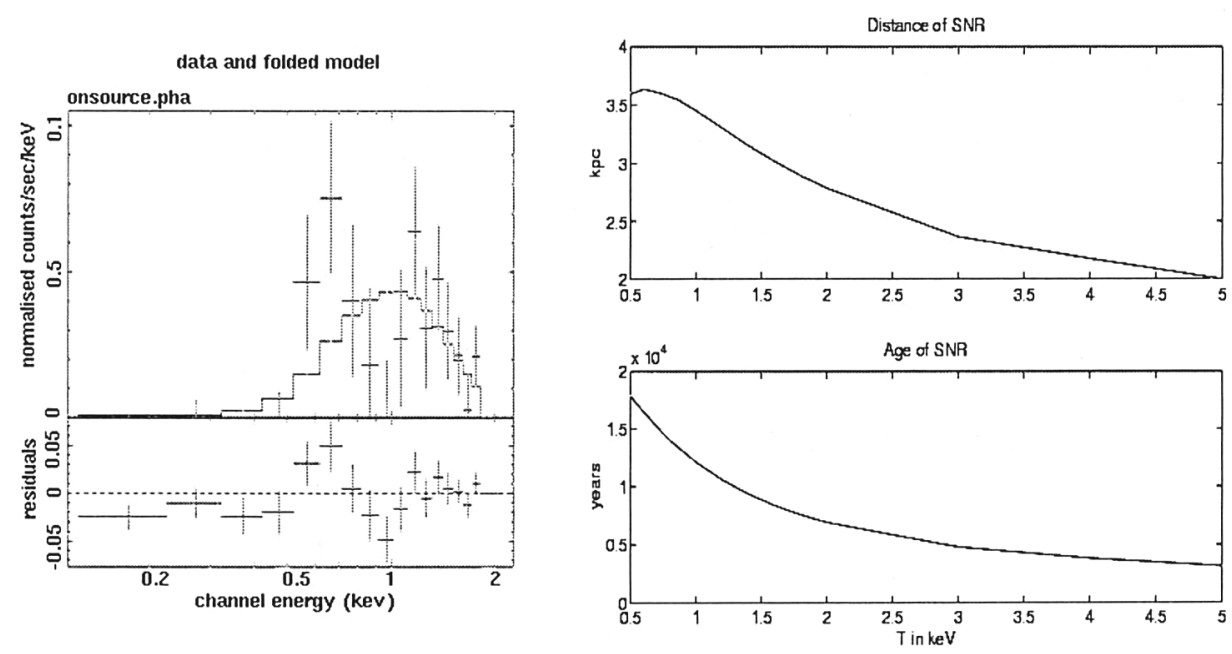

Figure 2. ROSAT residual counts, and fitted distance \& age model

\section{References}

R. Dodson. PhD thesis, Durham University, 1997.

http://kerr.phys.utas.edu.au/ rdodson/thesis.

D.A. Frail, W.M. Goss, and J.B.Z Whiteoak. Astrophys. J., 437(2):781-793, 1994.

Golap, K. and Issur, NH. and Somanah, R. and Dodson, R. and Modgekar, M. and Sachdev, S. and Shankar, N.U. and Sastry, C.V. Journal of Astrophysics and Astronomy 19:35-55, 1998.

S Johnston, et al. Mon. Not. R. astr. Soc., 255:401-411, 1992.

V. Kaspi. In Pulsars: Problems and Progress,IAU Colloquium 160, page 375. Astronomical Society of the Pacific, 1996.

B. Koribalski, S. Johnston, J.M. Weisberg, and W. Wilson. Astrophys. J., 441(2):756-764, 1995.

W.M. McAdam, J.L. Osborne, and M.L. Parkinson. Nature, 361:516-518, 1993.

L. Nicastro, S. Johnston, and B. Koribalski. Astron. Astrophys. Letters, 306:L49-L51, 1996. 